AperTO - Archivio Istituzionale Open Access dell'Università di Torino

\title{
Relevance of sample preparation for flow cytometry
}

\section{This is the author's manuscript}

Original Citation:

Availability:

This version is available http://hdl.handle.net/2318/1649593

since 2017-10-13T15:58:36Z

Published version:

DOI:10.1111/ijlh.12755

Terms of use:

Open Access

Anyone can freely access the full text of works made available as "Open Access". Works made available under a Creative Commons license can be used according to the terms and conditions of said license. Use of all other works requires consent of the right holder (author or publisher) if not exempted from copyright protection by the applicable law. 


\title{
Relevance of sample preparation for flow cytometry
}

\author{
V. E. Muccio | E. Saraci | M. Gilestro | D. Oddolo | M. Ruggeri | S. Caltagirone | \\ B. Bruno | M. Boccadoro | P. Omedè
}

Divisione Universitaria di Ematologia, A.O.U. Città della Salute e della Scienza di Torino,

Torino, Italy

\begin{abstract}
Introduction: Flow cytometry is a useful tool for diagnosis and minimal residual disease (MRD) study of hematological diseases. Standard sample preparation protocols are characterized by stain-lyse-wash (SLW). To prevent nonspecific bindings and achieve high sensitivity in MRD studies, lyse-wash-stain-wash (LWSW) is required. To our knowledge, no comparison between the two methods has been performed.

Methods: We compared mean fluorescence intensity (MFI), stain index, signal-tonoise ratio, and percentage of positive cells of 104 antibodies and of 13 selected antibodies tested in 10 samples simultaneously prepared with the two methods.

Results: MFI and percentages of positive cells obtained by the two methods did not show significant differences and showed a very high correlation. Stain index and signal-to-noise ratio presented higher values for kappa and lambda surface chains in LWSW samples and a trend of higher values for the other antibodies in SLW samples.

Conclusions: We suggest to use LWSW method also at diagnosis to obtain more comparable antibody intensity expressions when samples from the same patient are processed for MRD evaluation after bulk lysis. Moreover, LWSW can prevent nonspecific bindings, shows no differences in the identification and quantitation of the populations of interest, and reduces acquisition of cell debris.
\end{abstract}

\section{KEYWORDS}

antibody binding, flow cytometry, lyse-wash-stain, sample preparation, stain-lyse-wash

\section{1 | INTRODUCTION}

Over the past decades, multiparameter flow cytometry has become the method of choice for the differential diagnosis of several hematological diseases, for the definition of prognostic factors, and for the identification of rare cell populations in peripheral blood (PB) and bone marrow samples (BM). ${ }^{1-6}$

Flow cytometry, through the presence or absence of specific surface antigens, detects abnormal cells and helps in identifying their lineage and maturation stage, detects abnormal cells through identification of antigen expression that differs from the normal. The wider application of flow cytometry in diagnostic and research fields increases the need to distinguish between real and artifact positive signals given that antigen-antibody interactions, characterized by weak bindings, may involve unexpected molecules. The presence of dead cells, cell doublets, and the nonspecific antibody binding can compete to generate artifacts in the phenotype. In some cases, an antibody can bind similar antigens not of interest or an epitope can be shared by different antigens or the Fc of many antibodies can be bound by Fc-receptors expressed by different cells of the immune system. ${ }^{7}$ Undesired antibody's binding depends not only on the antibody's specificity but also on a wide variety of other interactions, as well as the physical and biological conditions that the target cell population encountered during the assay.

The ability to reliably distinguish between positive and negative populations of cells is an essential aspect of clinical flow cytometry. 
Sample preparation is a key phase to prevent nonspecific bindings or artifacts. Washing samples with a saline solution added with albumin before staining or treating them with Fc-block reagents reduce interferences in antibodybinding. ${ }^{8}$

A large number of guidelines suggest as standard preparation protocols for the immunophenotype study the stain-lyse-wash (SLW) methods to obtain best signal discrimination. ${ }^{9-17}$ However, many reports on minimal residual disease (MRD) studies by flow cytometry ${ }^{18-22}$ recommend a bulk lysis of the sample, which consists on the lysis of a high number of cells in PB or BM samples followed by washing and staining to increase sensitivity.

In this study, we compared the mean fluorescence intensity (MFI), stain index, signal-to-noise ratio, and percentages of positive cells tested with 104 surface antibodies before (SLW) and after lyse and wash (LWSW). Moreover, we compared the above-mentioned parameters with 13 selected antibodies used to identify monocyte and lymphocyte subpopulations.

\section{2 | MATERIALS AND METHODS}

\section{1 | Samples collection}

From August 2015 to January 2016, a total of 15 PB and 5 BM samples, collected in vacutainers containing EDTA (Becton Dickinson (BD) San Josè, California, USA), were obtained from patients sent to our laboratory for routine flow cytometry analysis and tested.

\section{2 | Ethics statement}

The data collection and additional analyses, such as statistical tests, were performed anonymously. This study was carried out in accordance with Declaration of Helsinki and subsequent amendments.

\section{3 | Lyse-wash-stain-wash (LWSW) procedure}

$1 \times 10^{6}$ of bone marrow cells or $100 \mu \mathrm{L}$ of peripheral blood is incubated in the darkness at room temperature (RT) for 10 minutes with $3 \mathrm{~mL}$ of $\mathrm{NH} 4 \mathrm{Cl}$ lysing solution ( $\mathrm{pH} \mathrm{7.3),} \mathrm{centrifuged} \mathrm{at} 4^{\circ} \mathrm{C}$ for $5 \mathrm{~min}-$ utes at $500 \mathrm{~g}$. The supernatant is discarded and the sample washed in $3 \mathrm{~mL}$ of phosphate saline buffer solution (PBS) + bovine serum albumin (BSA) $0.5 \%$ and sodium azide $0.1 \%\left(1 \mathrm{~mol} \mathrm{~L}^{-1}\right)$, stained with the appropriate amount of antibodies in darkness at RT for 15 minutes and washed in PBS-BSA.

\subsection{Stain-lyse-wash (SLW) procedure}

$1 \times 10^{6}$ of bone marrow cells or $100 \mu \mathrm{L}$ of peripheral blood is stained with the appropriate amount of antibodies in the darkness at RT for 15 minutes, incubated in the darkness at RT for 10 minutes with $3 \mathrm{~mL}$ of $\mathrm{NH} 4 \mathrm{Cl}$, centrifuged at $4^{\circ} \mathrm{C}$ for 5 minutes at $500 \mathrm{~g}$. The supernatant is discarded and the sample is washed in PBS-BSA.

Mean fluorescence intensity (MFI), stain index, signal-to-noise ratio, and percentages of positive cells were assessed by correlating the population positive for a given antibody with white blood cells in the sample gated by physical cytometric parameters.

To compare the 2 preparation methods, the same amount of antibodies was employed, and the same compensation was applied.

The 104 antibodies were studied as single reagent in a total of five BM and five PB samples for one replicate for each reagent. Their fluorochromes, clones, manufacturers, and SLW/LWSW MFI ratio are reported in Table1.

A 100.000 total events for each sample were acquired with Navios flow-cytometer Beckmann Coulter (BC) and data analysis was performed with Kaluza software (BC).

Unstained cells and single fluorochrome stained cells were used to set the instrument. Instrument performance was checked daily by recording fluorescence intensity with calibrating beads (Flow-Check Pro Fluorospheres BC, Beckman Coulter).

Moreover, 10 peripheral blood samples treated with both methods were tested with the following selected antibodies: CD45 krome orange (KO), CD14 phycoerythrin cyanine 5.5 (PC 5.5), HLA-DR fluorescein isothiocyanate (FITC), CD3 phycoerythrin cyanine 7 (PC-7), CD4 pacific blue (PB), CD8 allophycocyanin (APC), CD16 + CD56 phycoerythrin (PE), CD19 PC-7, CD20 APC-Alexa Fluor 750, CD5 PB, CD10 PC5.5 (BC), Lambda PE, Kappa FITC (DAKO).

In the analysis, the first step was to select white blood cells without debris by a FSC/SSC dot plot. The population well separated by all other cells, with a positive signal for an antigen (more than 10log of positivity), is selected as described in Figure 1.

The arithmetic MFI of each event for a given antibody was calculated by Kaluza software after the selection of the positive cell population.

Signal-to-noise ratio was calculated as follows: MFI of positive peak/MFI of negative peak and stain index as (MFI of positive peak - MFI of negative peak)/2*standard deviation of negative peak.

\section{5 | Statisticalanalysis}

Differences between means were studied by $T$ test; correlation between results was assessed by Pearson product-moment correlation coefficient, and agreement between two methods was determined by Bland-Altman plot.

\section{3 | RESULTS}

Means of MFI and of percentages of positive cells obtained by the two methods were not significantly different: mean MFI of SLW and LWSW were 27 and 29, respectively ( $P=.224)$, while mean $\%$ of SLW and LWSW were $20.4 \%$ and $20.7 \%(P=.186)$, respectively.

MFI and percentages obtained by the two methods showed a statistically significant correlation: $r^{2}=0.83 P<.001$ and $r^{2}=0.99$ $P<.001$, respectively (Figure $2 \mathrm{~A}$ and $\mathrm{B}$ ).

The Figure $2 \mathrm{~A}$ shows some antibodies with a MFI higher in LWSW than in SLW samples (indicated as white circles) that were as follows: CD235a FITC, CD235a PE, CD25 APC, CD14 PC5.5, 
TABLE 1 Antibodies, fluorochromes, and clones tested in the study

\begin{tabular}{|c|c|c|c|c|c|c|c|c|c|}
\hline Antibody & Fluorochrome & Company & Clone & SLW/LWSW MFI & Antibody & Fluorochrome & Company & Clone & SLW/LWSW MFI \\
\hline CD25 & APC & $\mathrm{BC}$ & B9E9 & 0.03 & CD4 & PB & $\mathrm{BC}$ & $13 \mathrm{~B} 8.2$ & 1 \\
\hline CD235a & PE & DAKO & SC159 & 0.04 & CD33 & PE & BC & 906 & 1 \\
\hline CD235a & FITC & DAKO & JC159 & 0.05 & CD4 & FITC & DAKO & MT310 & 1.01 \\
\hline Kappa/Lambda & FITC/PE & DAKO & Polyclonal & $0.35 / 0.16$ & CD117 & APC & CYTOGNOS & $104 D 2$ & 1.01 \\
\hline CD14 & PC5.5 & $\mathrm{BC}$ & RM052 & 0.36 & CD64 & PB & $B C$ & 22 & 1.03 \\
\hline CD11b & APC & $\mathrm{BD}$ & D12 & 0.42 & CD43 & FITC & DAKO & DF-T1 & 1.03 \\
\hline CD23 & PC-7 & $\mathrm{BD}$ & M-L233 & 0.47 & CD8 & APC & $\mathrm{BC}$ & SK1 & 1.04 \\
\hline CD66 & FITC & SANQUIN & CLB-B13.9 & 0.49 & CD45 & APC-H7 & BD & 2D1 & 1.05 \\
\hline CD71 & APC & $\mathrm{BD}$ & L01.1 & 0.55 & CD5 & PB & $\mathrm{BC}$ & BL1a & 1.05 \\
\hline CD15 & PerCP-Сy5.5 & $\mathrm{BD}$ & HI98 & 0.56 & $\mathrm{CD} 38$ & PC-7 & $\mathrm{BD}$ & HB7 & 1.05 \\
\hline CD52 & PE & INVITROGEN & CF1D12 & 0.57 & CD20 & APC-A700 & $\mathrm{BC}$ & B9E9 & 1.06 \\
\hline CD33 & FITC & $\mathrm{BC}$ & D3HL60.251 & 0.6 & CD14 & APC & BD & $\mathrm{M} \phi \mathrm{P} 9$ & 1.07 \\
\hline CD13 & PE & $\mathrm{BC}$ & SJ1D1 & 0.64 & CD20 & PC-7 & $\mathrm{BD}$ & L27 & 1.07 \\
\hline CD38 & APC-A750 & $\mathrm{BC}$ & LS198-4-3 & 0.67 & CD200 & APC-A750 & $\mathrm{BC}$ & OX-104 & 1.11 \\
\hline CD20 & PE & DAKO & B-LY1 & 0.69 & CD4 & PC7 & BD & SK3 & 1.13 \\
\hline CD10 & PC5.5 & $\mathrm{BC}$ & ALB1 & 0.71 & CD45 & V450 & $B D$ & HI30 & 1.13 \\
\hline CD16 & PerCP-Сy5.5 & $\mathrm{BD}$ & $3 \mathrm{G} 8$ & 0.74 & CD8 & PC-7 & $\mathrm{BD}$ & SK1 & 1.14 \\
\hline CD14 & FITC & DAKO & TüK4 & 0.74 & $\mathrm{CD} 22$ & PE & $\mathrm{BD}$ & S-HCL-1 & 1.16 \\
\hline CD14 & PC-7 & $\mathrm{BD}$ & M5E2 & 0.76 & CD3 & V450 & BD & UCHT1 & 1.17 \\
\hline CD138 & PB & $B C$ & B-A38 & 0.77 & CD8 & PerCP & BD & SK1 & 1.17 \\
\hline CD64 & FITC & BIOLEGEND & 10.1 & 0.79 & CD20 & APC-A750 & $B C$ & B9E9 & 1.17 \\
\hline CD138 & PC5.5 & $\mathrm{BC}$ & B-A38 & 0.82 & CD14 & APC-H7 & BD & $\mathrm{M} \phi \mathrm{P} 9$ & 1.18 \\
\hline CD33 & PC5.5 & $B C$ & D3HL60.251 & 0.83 & CD34 & FITC & DAKO & BIRMA-K3 & 1.2 \\
\hline CD10 & APC & $\mathrm{BD}$ & HI10a & 0.84 & CD71 & FITC & DAKO & Ber-T9 & 1.2 \\
\hline CD33 & APC & $B C$ & D3HL60.251 & 0.84 & CD20 & FITC & $\mathrm{BD}$ & L27 & 1.22 \\
\hline CD4 & APC-H7 & BD & SK3 & 0.84 & CD45 & APC & $\mathrm{BD}$ & 2D1 & 1.24 \\
\hline CD22 & FITC & BD & S-HCL-1 & 0.85 & CD4 & PerCP-Сy5.5 & $\mathrm{BD}$ & SK3 & 1.26 \\
\hline CD34 & APC & $\mathrm{BC}$ & 581 & 0.86 & CD71 & APC-A700 & $\mathrm{BC}$ & YDJ1.2.2 & 1.26 \\
\hline CD45 & KO & $\mathrm{BC}$ & $\mathrm{J} .33$ & 0.88 & CD5 & PC-7 & $\mathrm{BD}$ & L17F12 & 1.29 \\
\hline CD79B & PE & DAKO & SN8 & 0.88 & CD34 & PC-7 & $\mathrm{BD}$ & $8 \mathrm{G} 12$ & 1.29 \\
\hline CD16 & PE & $B C$ & $3 \mathrm{G} 8$ & 0.9 & HLA-DR & PE & DAKO & AB3 & 1.3 \\
\hline CD23 & APC & $B C$ & $9 \mathrm{P} 25$ & 0.9 & CD7 & APC & $\mathrm{BD}$ & M-T701 & 1.31 \\
\hline
\end{tabular}




\begin{tabular}{|c|c|c|c|c|c|c|c|c|c|}
\hline Antibody & Fluorochrome & Company & Clone & SLW/LWSW MFI & Antibody & Fluorochrome & Company & Clone & SLW/LWSW MFI \\
\hline CD59 & PE & INVITROGEN & MHCD5904 & 0.9 & CD20 & PerCP-Cy5.5 & $\mathrm{BD}$ & L27 & 1.33 \\
\hline CD4 & V450 & $\mathrm{BD}$ & RPA-T4 & 0.9 & CD22 & PC5.5 & $\mathrm{BC}$ & $\mathrm{SJ} 10.1 \mathrm{H} 11$ & 1.33 \\
\hline CD38 & APC & $\mathrm{BC}$ & LS198-4-3 & 0.92 & CD71 & APC-H7 & $\mathrm{BD}$ & M-A712 & 1.37 \\
\hline CD5 & FITC & $\mathrm{BC}$ & BL1a & 0.92 & CD15 & PE & $\mathrm{BC}$ & $80 \mathrm{H} 5$ & 1.38 \\
\hline HLA-DR & APC & $\mathrm{BC}$ & IMMU-357 & 0.93 & CD19 & APC & $\mathrm{BC}$ & J3-119 & 1.4 \\
\hline CD45 & V500 & $\mathrm{BD}$ & HI30 & 0.94 & CD3 & APC & IMMUNOSTEP & $145-2 \mathrm{C} 11$ & 1.4 \\
\hline CD56 & PE & $\mathrm{BC}$ & N901 & 0.95 & CD45 & PerCP-Cy5.5 & $\mathrm{BD}$ & 2D1 & 1.42 \\
\hline CD117 & PE & CYTOGNOS & 104D2 & 0.95 & CD3 & PE & DAKO & UCHT1 & 1.44 \\
\hline HLA DR & FITC & $\mathrm{BC}$ & B8.12.2 & 0.96 & CD14 & PE & DAKO & TöK4 & 1.44 \\
\hline CD138 & APC & $\mathrm{BD}$ & MI15 & 0.96 & CD138 & FITC & CYTOGNOS & B-A38 & 1.52 \\
\hline CD3 & FITC & DAKO & UCHT1 & 0.97 & CD8 & APC-H7 & $\mathrm{BD}$ & SK1 & 1.52 \\
\hline CD38 & PB & $\mathrm{BC}$ & LS198-4-3 & 0.97 & CD5 & PE & $\mathrm{BC}$ & BL1a & 1.53 \\
\hline CD5 & PC5.5 & $\mathrm{BC}$ & BL1a & 0.97 & CD34 & PE & $\mathrm{BD}$ & $8 \mathrm{G} 12$ & 1.54 \\
\hline CD20 & APC & $\mathrm{BC}$ & B9E9 & 0.97 & CD3 & PC7 & $\mathrm{BC}$ & UCHT1 & 1.58 \\
\hline CD55 & PE & $\begin{array}{r}\text { Southern } \\
\text { Biotech }\end{array}$ & $143-30$ & 0.99 & $\mathrm{CD} 23$ & PE & DAKO & MHM6 & 1.59 \\
\hline CD56 & APC & $B C$ & N901 & 0.99 & $\mathrm{CD} 24$ & PE & $\mathrm{BD}$ & ML5 & 1.61 \\
\hline CD56 & V450 & $\mathrm{BD}$ & B159 & 1 & CD33 & PC-7 & $\mathrm{BD}$ & P67.6 & 1.86 \\
\hline CD19 & PB & $\mathrm{BC}$ & J3-119 & 1 & $\mathrm{CD} 2$ & PC-7 & $\mathrm{BC}$ & $39 C 1.5$ & 1.89 \\
\hline CD19 & PC-7 & $\mathrm{BC}$ & J3-119 & 1 & CD45 & PB & DAKO & T29/33 & 2.47 \\
\hline
\end{tabular}

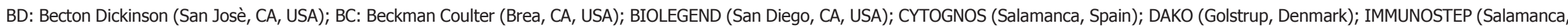
Spain); INVITROGEN (Waltham, MA, USA); SANQUIN (Amsterdam, The Nederlands); Southern Biotech (Birmingham, AL, USA).

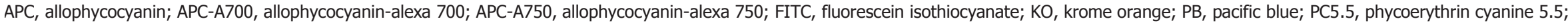

$\mathrm{PC}-7$, phycoerythrin cyanine 7; $\mathrm{PE}$, phycoerythrin. 
(A)
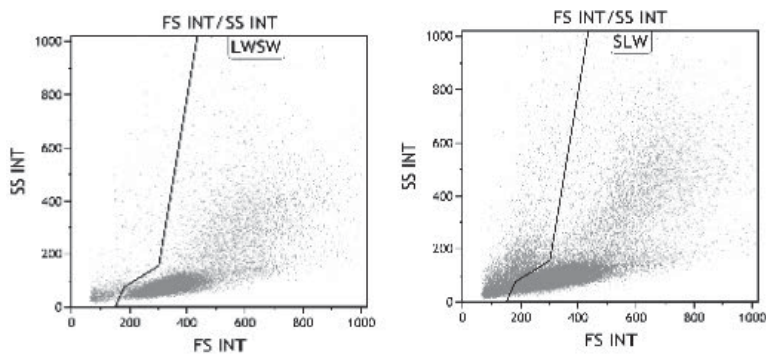

(B)
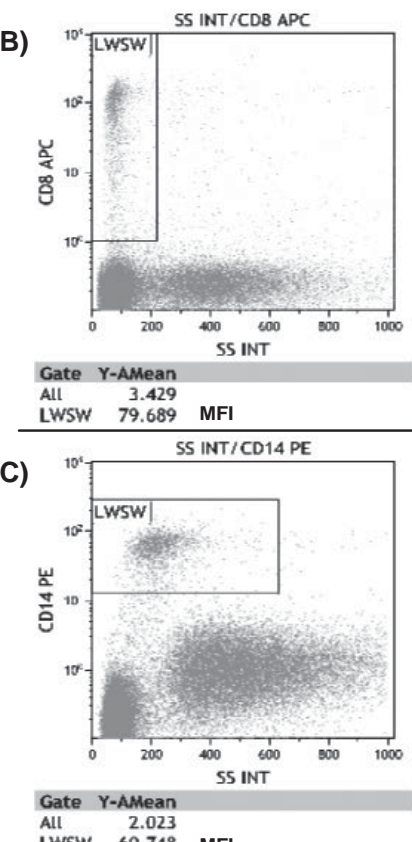

\begin{tabular}{lr} 
All & 2.023 \\
LWSW & 60.748 \\
\hline
\end{tabular}

(D)
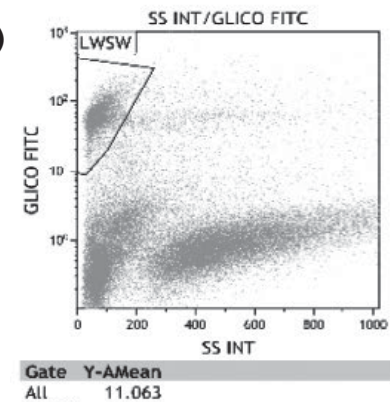

LWSW 66.885 MFI

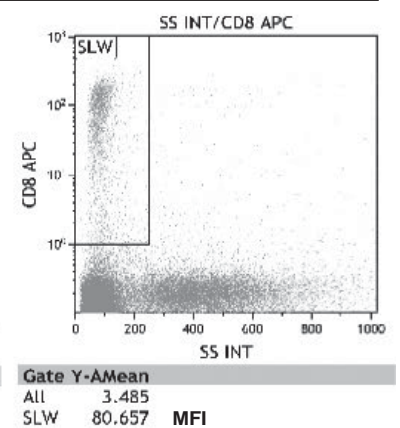

SLW 80.657 MF

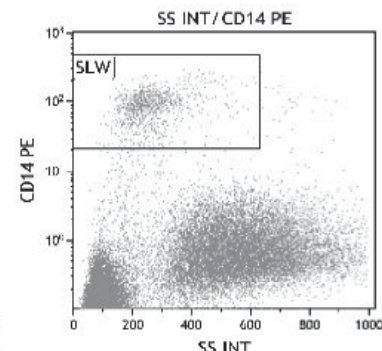

Gate Y-AMean

$\begin{array}{lrl}\text { All } & 2.223 & \\ \text { SLW } & 92.589 & \text { MFI }\end{array}$

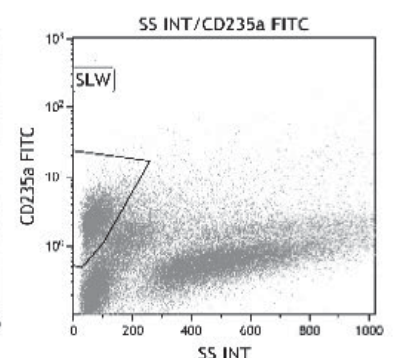
$\begin{array}{ll}\text { All } & 1.440 \\ \text { SLW } & 2.762 \quad \mathrm{MFI}\end{array}$
Gate Y-AMean

FIGURE 1 Gating strategy and exemplificative dot plots. (A) physical parameters obtained by the two methods. (B) antibody with similar MFI obtained by the two methods. (C) antibody with higher MFI obtained by SLW than LWSW method. (D) antibody with higher MFI obtained by LWSW than SLW method

CD11b APC, and CD71 APC, while the antibodies with higher MFI in SLW than LWSW sample were CD57 FITC and CD14 PE (indicated as white squares).

Bland-Altman analysis of percentages of positive cells showed mean difference of -0.20 with standard deviation of 2.04 , and all differences between SLW and LWSW values were within a range of $\pm 5 \%$ (Figure 3).

Bland-Altman analysis of MFI of positive cells showed mean of differences of -2.14 with standard deviation of 17.9 (data not shown).
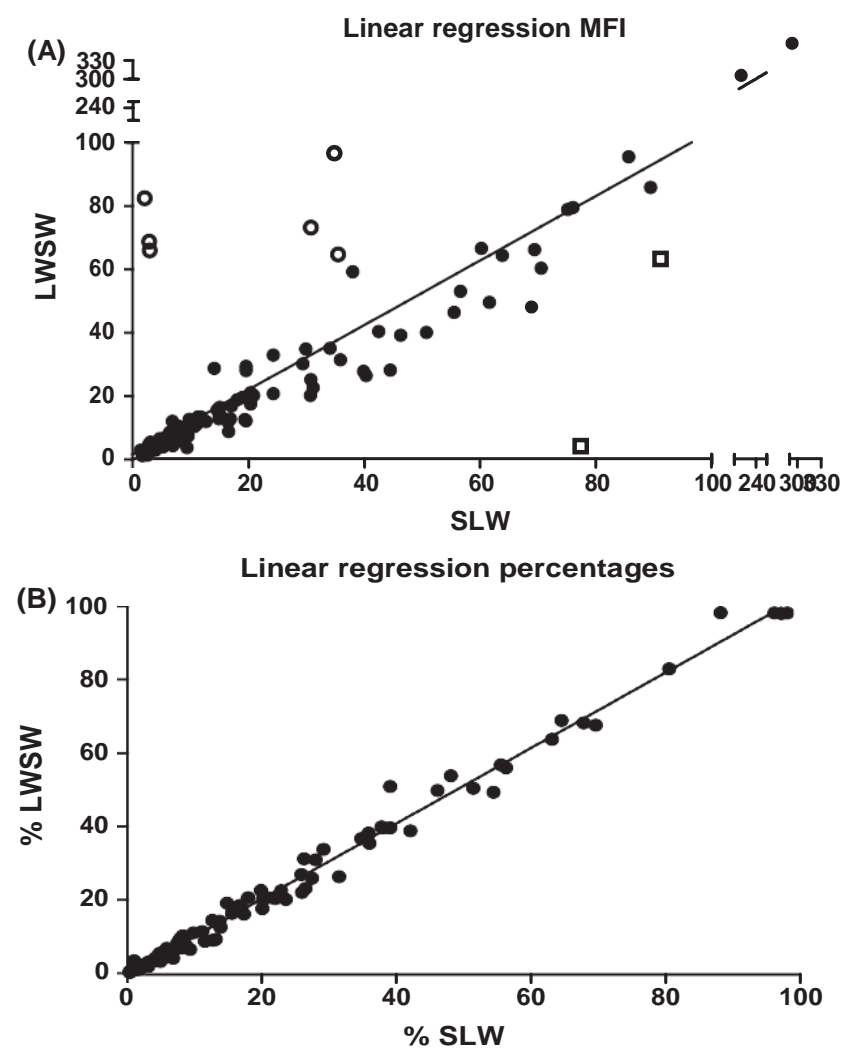

FIGURE 2 Linear regression of MFI and percentages values of 104 antibodies in samples simultaneously prepared with LWSW and SLW methods. (A) Linear regression of MFI values of 104 antibodies in samples simultaneously prepared with LWSW and SLW methods. White circles represent samples with visible higher MFI in LWSW than in SLW samples. White squares represent samples with visible higher MFI in SLW than in LWSW samples. (B) Linear regression of percentage of cells positive for 104 antibodies in samples simultaneously prepared with LWSW and SLW methods

Signal-to-noise ratio and stain index were higher in SLW than LWSW samples (59.8 vs $41.7 P<.001,24.4$ vs $17.8 P=.001$, respectively).

Means of debris obtained by SLW (31.8\%) and LWSW (19.3\%) were significantly different $P<.001$ ) (Figure 4).

Analysis of 13 selected antibodies tested in 10 samples showed:

- no statistical significant differences in the percentages of positive cells;

- higher MFI for CD3 (means: 30.6 vs $23.6 P<.01$ ) in SLW than LWSW samples and for HLA-DR (means: 12.3 vs $27.1 P<.05$ ), CD16 + 56 (means: 74.4 vs $123.6 P<.01$ ) in LWSW than SLW samples;

- higher signal-to-noise ratio in SLW samples for CD45 (means: 51.6 vs $36.6 P<.05$ ), CD3 (means: 230.8 vs $36.9 P<.05)$, CD19 (means: 92.8 vs $46.8 P<.01$ ), and CD10 (means: 49.7 vs $20.6 P<.01$ );

- higher stain index in SLW than LWSW samples for CD3 (means: 57.9 vs $20.2 P<.01$ ), CD19 (means: 30.1 vs $20.9 P<.01$ ), and CD10 (means: 28.4 vs $14.81 P<.01$ );

- higher MFI, stain index, and signal-to-noise ratio in LWSW samples 


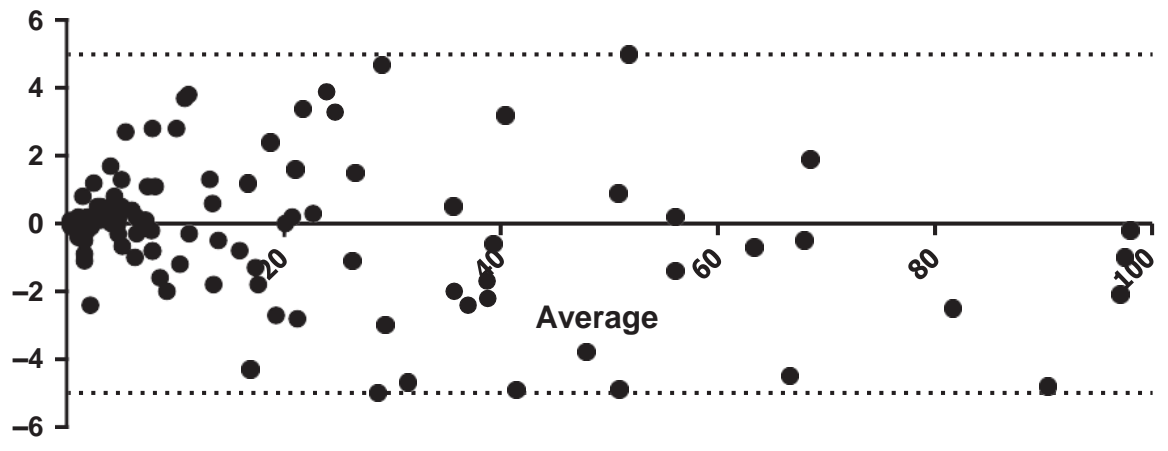

FIGURE 3 Bland-Altman analysis of percentages of cells positive for 104 antibodies in samplessimultaneously prepared with LWSW and SLW methods

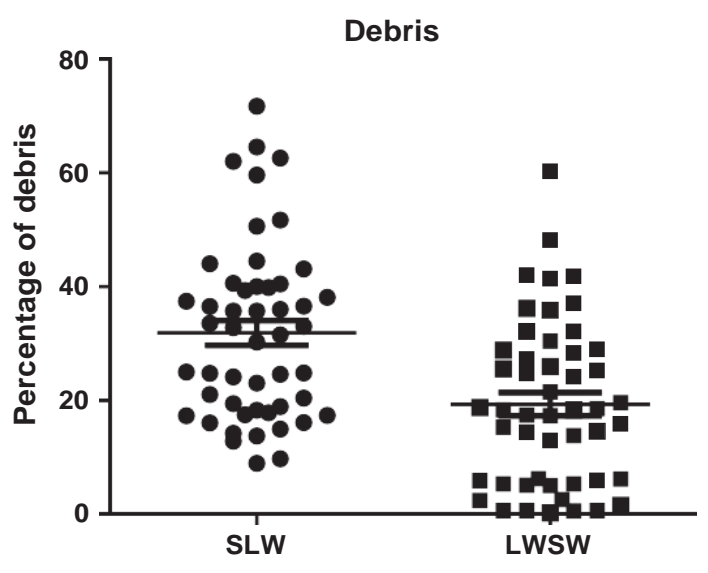

FIGURE 4 Percentages of debris obtained in LWSW and SLW samples

for surface kappa and lambda chains (means MFI: kappa LWSW 18.9 vs kappa SLW 7.4 P < .05; lambda LWSW 30.8 vs lambda SLW $2.1 P<.01$; means stain index: kappa LWSW 8.8 vs kappa SLW 4.2 $P<.01$; lambda LWSW 14.6 vs lambda SLW $7.6 P<.05$, means signal-to-noise ratio: kappa LWSW 22 vs kappa SLW $7.6 P<.05$; lambda LWSW 33.3 vs lambda SLW 12 P $<.05$ ).

\section{4 | CONCLUSION}

Different methods to prepare samples for flow cytometry analysis have been described in a high number of study groups, and the SLW method is considered the standard one, because it provides the best signal output. However, in the antigen-antibody interactions, some difficulties can occur for the interactions with similar epitopes or for the interferences of different cells of immune system that can bind the Fc with a specific Fc-receptor. ${ }^{7}$ A solution for this problem, in particular for the study of surface kappa and lambda chains, is offered by some study groups that recommended the sample washing with PBS added with BSA to remove interfering free extracellular immunoglobulins ${ }^{23}$ or to avoid artifacts of cytophilic antibodies. ${ }^{9}$ Therefore, lyse and wash of a sample before staining, as in LWSW method, can prevent artifacts and, in MRD studies, allows to obtain a higher number of cells. To our knowledge, no comparison between these two methods has been described and it is not known whether a second wash gives significant differences in terms of antigens' binding and expression. We compared the expression of 104 antibodies assessed by both SLW and LWSW to identify differences between the two methods. Samples prepared with both methods did not show significant differences in terms of percentage and MFI of positive cells even though stain index and signal-to-noise ratio were significantly higher in SLW than LWSW samples. The antibodies with higher MFI in LWSW method, indicated as white circles in Figure 2A (CD235a FITC, CD235a PE, CD25 APC, CD14 PC5.5, CD11b APC, and CD71 $A P C)$, showed an MFI increase that overcame the slight increase of background. In two cases, indicated as white squares (CD57 FITC and CD14 PE), this phenomenon was observed in SLW samples. Moreover, the LWSW method increased both the intensity of background and of positive signal, but the stain index was reduced because the background exceeded the signal. However, the selection of positive cell populations was not affected by these differences, and no different percentages were obtained.

Mean of differences between SLW and LWSW percentages was -0.20 , and all differences were within a range of $\pm 5 \%$. Moreover, SLW samples presented a significantly higher percentage of debris than LWSW samples that determined higher number of "useful events" with the same threshold setup.

We also analyzed the expression of 13 selected antibodies in 10 samples prepared with the two methods. No significant difference in terms of percentage of positive cells was observed and, except for surface kappa and lambda chains, all other antibodies have shown higher values of stain index and signal-to-noise ratio in SLW samples than LWSW.

Despite the high concordance of MFI between the two methods, the signal-to-noise ratios and the stain indexes obtained, confirmed that SLW gave the best signal discrimination as reported by some guidelines $^{9-17}$ and that the expression of kappa and lambda surface immunoglobulins became brighter after sample washing. ${ }^{24}$

However, to achieve a high sensitivity for MRD evaluation, LWSW should be performed as suggested by the Euroflow consortium. ${ }^{22}$

We suggest the use of LWSW also in samples from newly diagnosed patients because, despite differences in stain index and signalto-noise ratio, the percentages of positive cells were similar in the two 
methods. Moreover, the use of the same method to stain cells from a patient at diagnosis and throughout the follow-up is recommended, to obtain more comparable cell parameters. Finally, LWSW, through the sample wash before the staining, prevents artifacts which may occur also in fresh samples and allows to acquire a higher number of useful events by reducing cell debris.

\section{ACKNOWLEDGEMENTS}

Authors thank the Fondazione Neoplasie Sangue Onlus for research support.

\section{CONFLICT OF INTEREST}

There authors declare no conflicts of interest.

\section{AUTHOR'S CONTRIBUTION}

VEM, ES, and MG designed the study, analyzed, and interpreted the data; DO, MR, and SC collected data; BB, MB, and PO drafted and approved the final version of manuscript.

\section{REFERENCES}

1. Marcondes NA, Fernandes FB, Faulhaber GA. Lineage determination in acute leukemias. Cytometry B Clin Cytom. 2014;86:149.

2. Shim YK, Rachel JM, Ghia P, et al. Monoclonal B-cell lymphocytosis in healthy blood donors: an unexpectedly common finding. Blood. 2014;123:1319-1326.

3. Kern W, Bacher U, Schnittger S, Alpermann T, Haferlach C, Haferlach T. Multiparameter flow cytometry reveals myelodysplasia-related aberrant antigen expression in myelodysplastic/myeloproliferative neoplasms. Cytometry B Clin Cytom. 2013;84:194-197.

4. Parikh SA, Kay NE, Shanafelt TD. Monoclonal B-cell lymphocytosis: update on diagnosis, clinical outcome, and counselling. Clin Adv Hematol Oncol. 2013;11:720-729.

5. Cao F, Zhao H, Li Y, Dai S, Wang C. Clinicopathological and phenotypic features of chronic NK cell lymphocytosis identified among patients with asymptomatic lymphocytosis. Int I Lab Hematol. 2015;37:783-790.

6. Paiva $B$, Vídriales $M B$, Rosiñol $L$, et al. A multiparameter flow cytometry immunophenotypic algorithm for the identification of newly diagnosed symptomatic myeloma with an MGUS-like signature and long-term disease control. Leukemia. 2013;27:2056-2061.

7. Hulspas R, O'Gorman MRG, Wood BL, Gratama JW, Sutherland DR. Considerations for the control of background fluorescence in clinical flow cytometry. Cytometry B Clin Cytom. 2009;76B:355-364.

8. Kuonen F, Touvrey C, Laurent J, Ruegg C. Fc block treatment, dead cells exclusion, and cell aggregates discrimination concur to prevent phenotypical artifacts in the analysis of subpopulations of tumor-infiltrating CD11b (+) myelomonocytic cells. Cytometry A. 2010;77A:1082-1090.

9. Davis BH, Wood B, Oldaker T, Barnett D. Validation of cell-based fluorescence assays: practice guidelines from the ICSH and ICCS. Cytometry B Clin Cytom. 2013;84B:282-285.

10. Macey MG, McCarthy DA, Milne T, Cavenagh JD, Newland AC. Comparative study of five commercial reagents for preparing normal and leukaemic lymphocytes for immunophenotypic analysis by flow cytometry. Cytometry. 1999;38:153-160.

11. Kraan J, Gratama JW, Haioun C, et al. Flow cytometric immunophenotyping of cerebrospinal fluid. Curr Protoc Cytom. 2008;6:25.

12. Stewart CC, Stewart SJ. Immunophenotyping. Curr Protoc Cytom. 2001;6:2.

13. Craig $\mathrm{FE}$, Ohori NP, Gorrill TS, Swerdlow SH. Flow cytometric immunophenotyping of cerebrospinal fluid specimens. Am J Clin Pathol. 2011;135:22-34.

14. Holmes K, Lantz LM, Fowlkes BJ, Schmid I, Giorgi JV. Preparation of cells and reagents for flow cytometry. Curr Protoc Immunol. 2001;5:3.

15. Greig B, Oldaker T, Warzynski M, Wood B. 2006 Bethesda International Consensus recommendations on the immunophenotypic analysis of hematolymphoid neoplasia by flow cytometry: recommendations for training and education to perform clinical flow cytometry. Cytometry B Clin Cytom. 2007;72(Suppl. 1):S23-S33.

16. Lee JA, Spidlen J, Boyce K, et al. MIFlowCyt: the minimum information about a Flow Cytometry Experiment. Cytometry A. 2008;73:926-930.

17. Kalina T, Flores-Montero J, van der Velden VH. EuroFlow standardization of flow cytometer instrument settings and immunophenotyping protocols. Leukemia. 2012;26:1986-2010.

18. Patkar N, Alex AA, Bargavi B, et al. Standardizing minimal residual disease by flow cytometry for precursor $B$ lineage acute lymphoblastic leukemia in a developing country. Cytometry B Clin Cytom. 2012;82:252-258.

19. Irving J, Jesson J, Virgo P, et al. Establishment and validation of a standard protocol for the detection of minimal residual disease in B lineage childhood acute lymphoblastic leukemia by flow cytometry in a multi-center setting. Haematologica. 2009;94:870-874.

20. Böttcher S, Stilgenbauer S, Busch R, et al. Standardized MRD flow and ASO IGH RQ-PCR for MRD quantification in CLL patients after rituximab-containing immunochemotherapy: a comparative analysis. Leukemia. 2009;23:2007-2017.

21. Westers TM, Ireland R, Kern W, et al. Standardization of flow cytometry in myelodysplastic syndromes: a report from an international consortium and the European LeukemiaNet Working Group. Leukemia. 2012;26:1730-1741.

22. EuroFlow Standard Operating Protocol (SOP) for Bulk Lysis for MRD panels (version 1.1 - 6 May 2014) by ESLHO and EuroFlow. http:// www.euroflow.org/usr/pub/prlogin.php [accessed 11 January 2016].

23. Johansson U, Bloxham D, Couzens S, Jesson J, Morilla R, Erber W. Guidelines on the use of multicolor flow cytometry in the diagnosis of haematological neoplasms. Br J Haematol. 2014;165: 455488.

24. ICCS e-Newsletter Vol. VI, No. 3, Summer 2015. http://www.cytometry.org/public/newsletters/eICCS-6-3/article2.php. 